tide.

\title{
CIRiS: Compact Infrared Radiometer in Space
} August, 2017

David Osterman

PI, CIRiS Mission Presented by Hansford Cutlip cal con 


\section{Overview of the CIRiS instrument and mission}

- The CIRiS instrument is a radiometric thermal infrared imaging instrument integrated to a $6 \mathrm{U}$ CubeSat spacecraft

- Three imaging bands from 7.5 um to 12.7 um

- CIRiS will be launched into Low Earth Orbit

- The mission objectives are to:

1. Demonstrate new technologies for high accuracy, on-orbit calibration compatible with Smallsats

2. Optimize radiometric calibration for science and operational applications

- The CIRiS instrument is modular, by design, to facilitate specialized implementations

- The design may be optimized for specific planetary science objectives

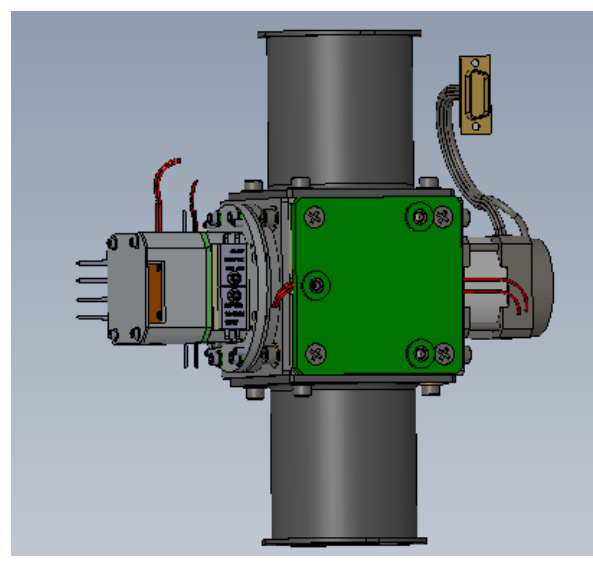

CIRiS instrument

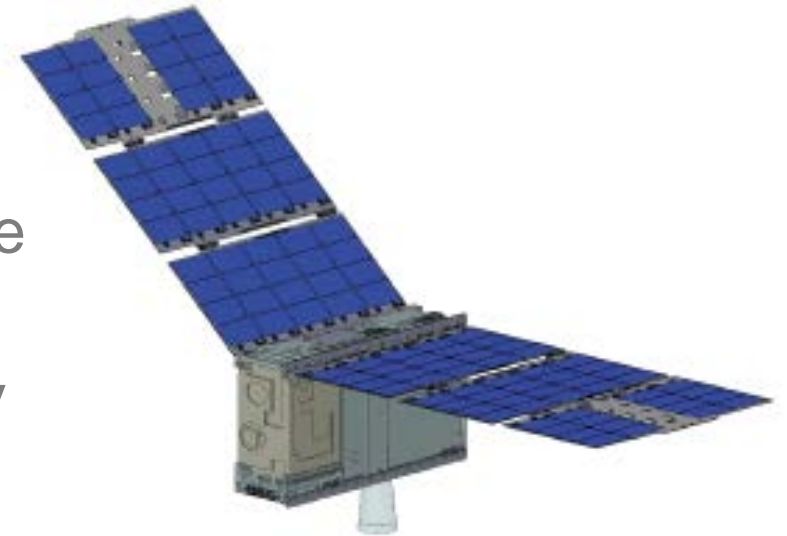

CIRiS spacecraft 


\section{Why radiometric imaging in the thermal infrared?}

Scientific and operational applications for Earth observations:

1. Land management

- Land surface temperature - analyze for soil moisture and drought impact

- Infrared reflectance- analyze for plant health and stress

2. Cloud microphysical effects for weather research

- Particle radius, thermodynamic phase, optical thickness

3. Validate climate models

- Local spatial and temporal variations in upwelling radiance, Earth's radiation imbalance

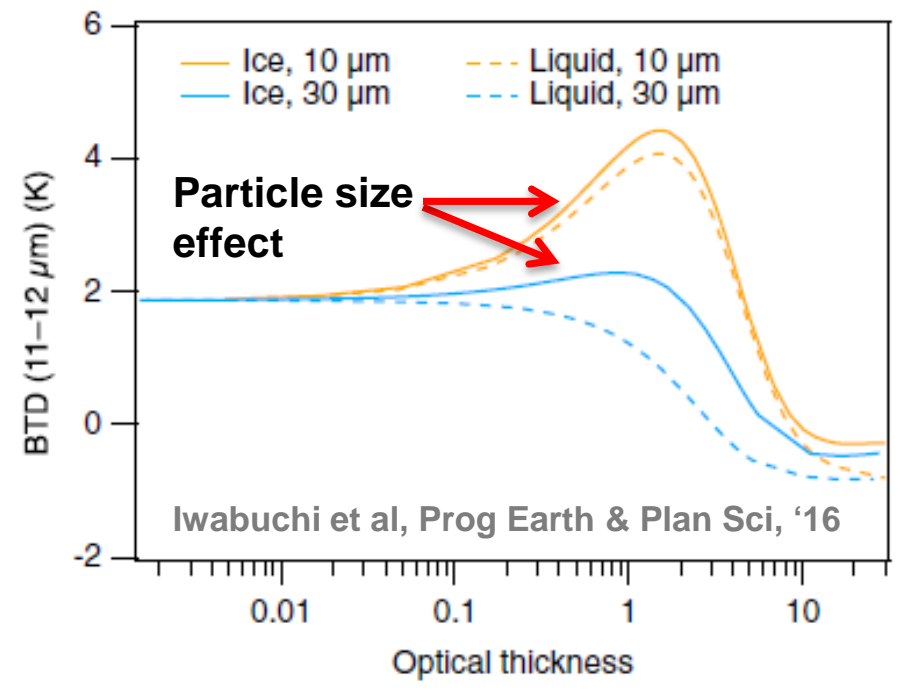

Applications in planetary science:

- Surface temperature, plumes, volcanism, tidal heating, ice fracturing and trapped liquid, particle size and compaction, mineralogy, global heat flux 
The CIRiS instrument adapts the design of a prior aircraft mounted Ball Aerospace instrument

- BESST: Ball Experimental Sea Surface Temperature Radiometer

- Used primarily as a remote radiometric thermal imager for Sea Surface Temperature

- Operated on aircraft and UAV campaigns

- A radiometric imager with two on-board blackbody sources
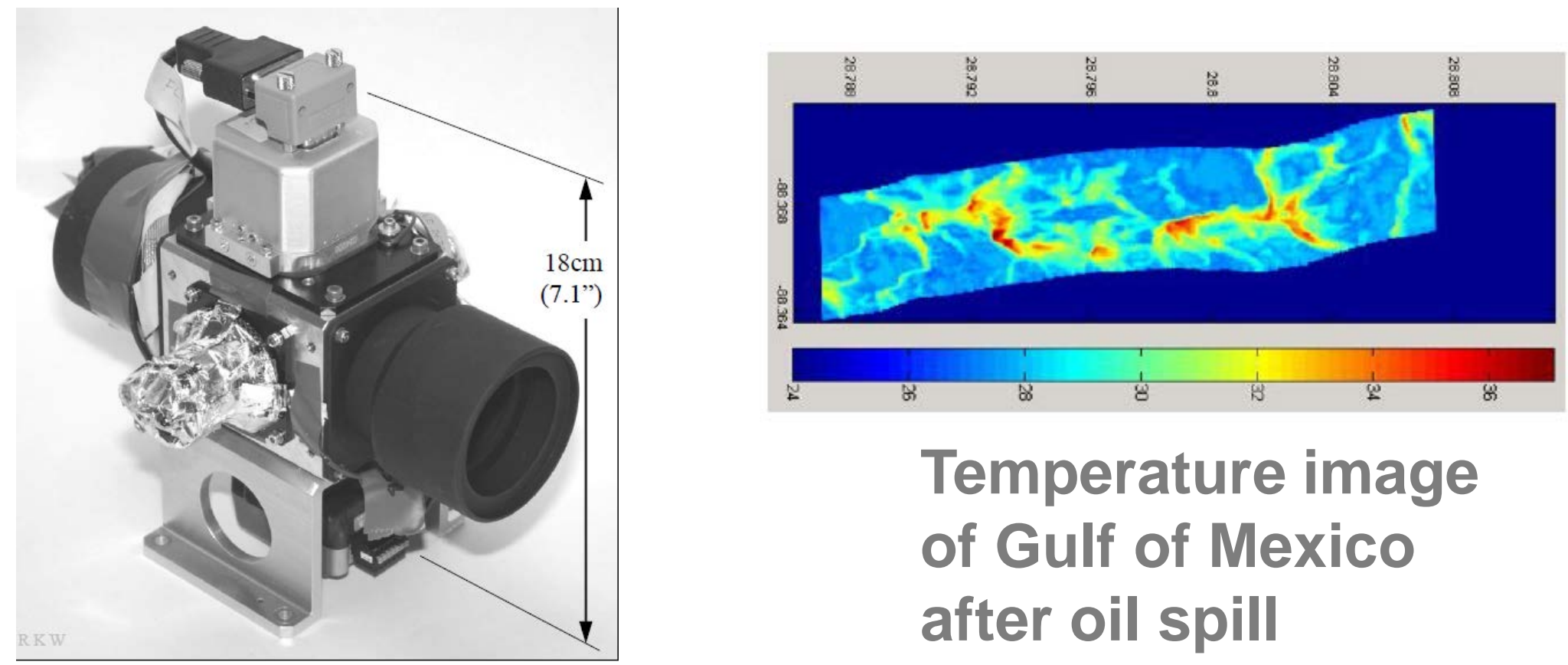

Temperature image of Gulf of Mexico after oil spill 


\section{The CIRiS guiding design objective is high radiometric accuracy in a compact envelope}

- CIRiS design features for high radiometric performance:

- Symmetric optomechanical structure to minimize calibration transfer offsets

- High emissivity (>>0.99) carbon nanotube blackbody sources

- Three calibration scenes

- End-to-end on-orbit calibration

- Knowledge and control of instrument component temperatures

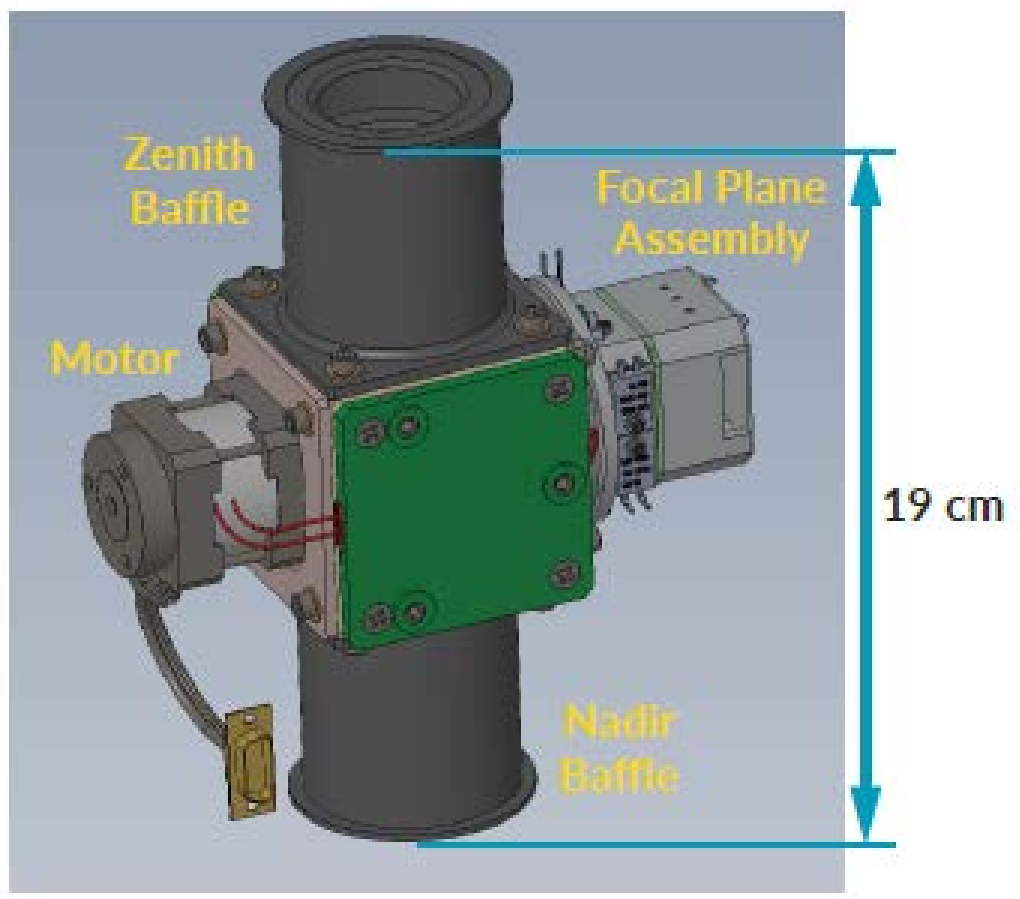




\section{The CIRiS scene-select mirror points the field of view}

\section{in one of four directions}

- Three calibration scenes, one science scene

- One source at on-board ambient temp: $280 \mathrm{~K}$

- One source at controlled temperature: $280 \mathrm{~K}$ to $300 \mathrm{~K}$

- View to deep space

- Four-fold symmetry minimizes background variation during transfer of calibration to science view

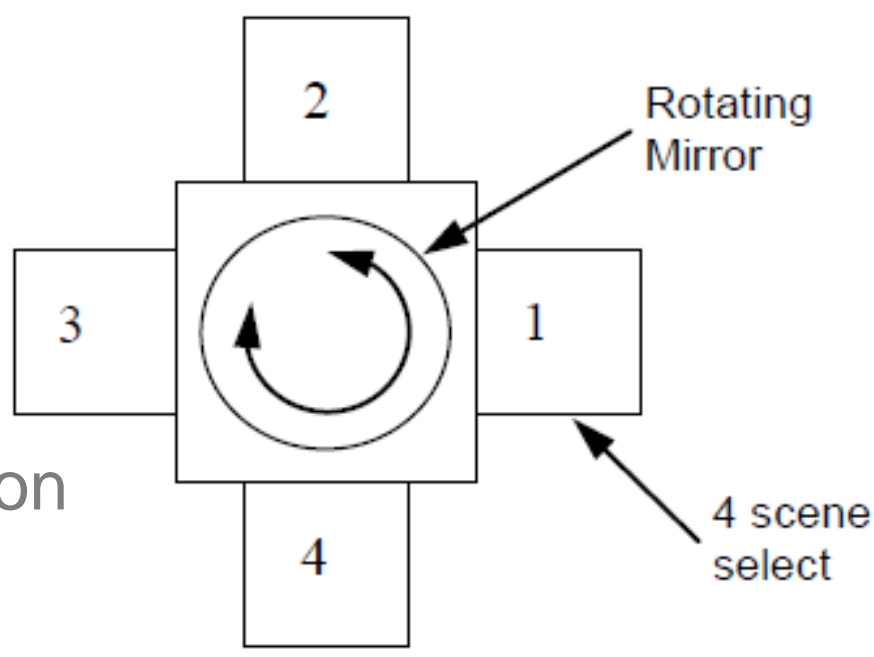

- Calibration is end-to-end: FPA to front aperture

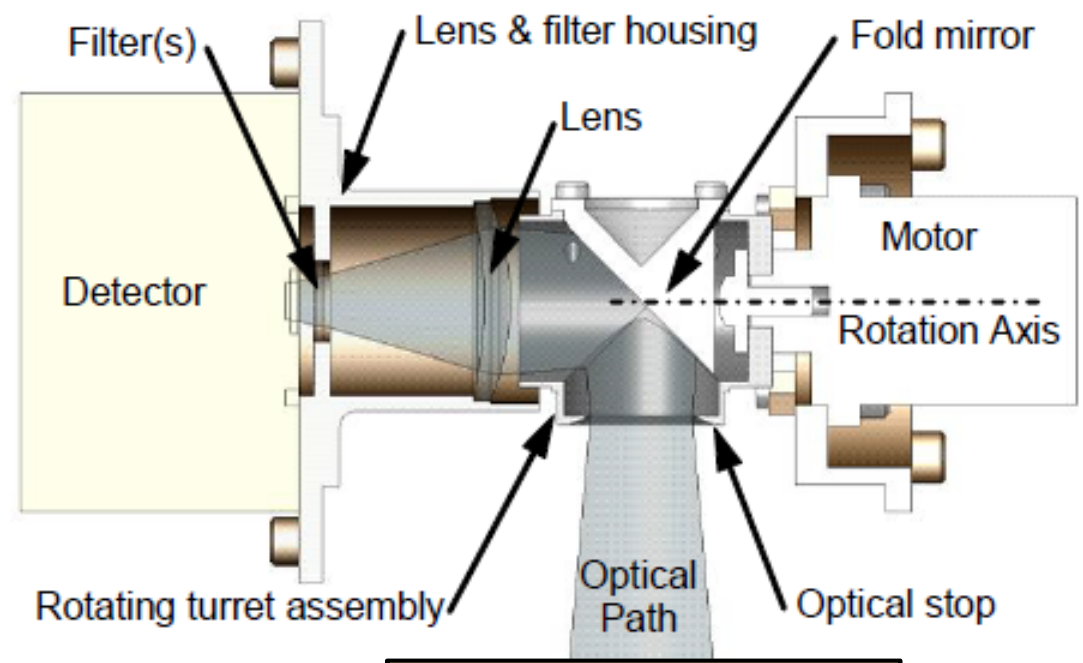

Blbdy or scene 
An enabling technology for high calibration performance in a small volume: Carbon Nanotube (CNT) sources

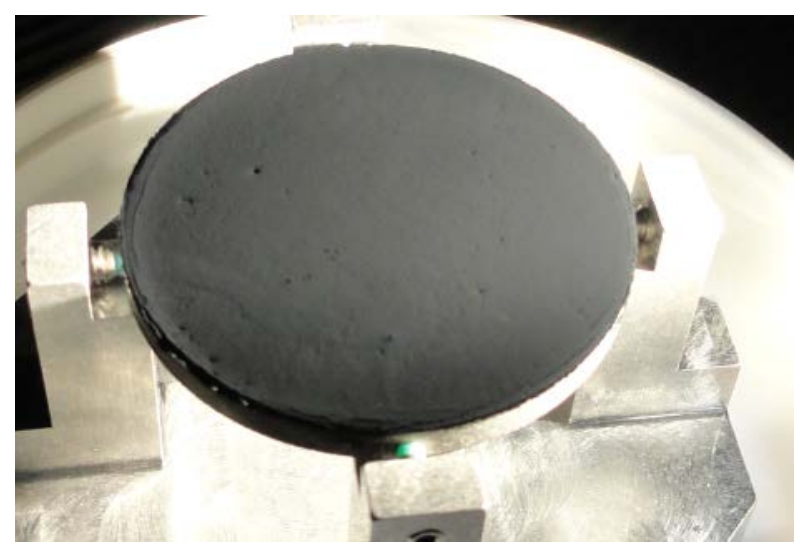

CIRiS flight sample, 2.5 in diameter

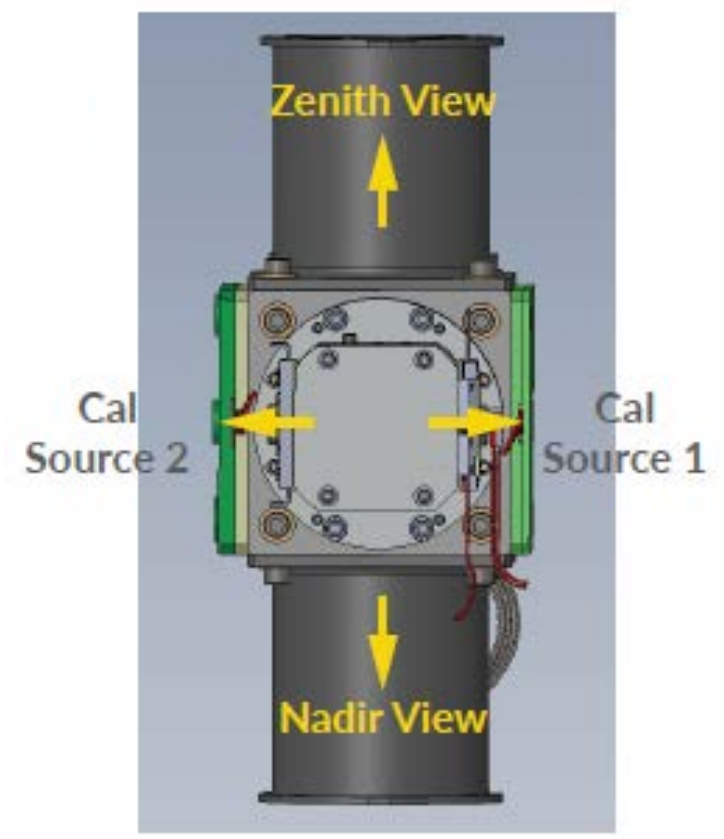

- CNT films on solid substrates are blackbody sources exhibiting very high emissivity in a much smaller volume then conventional cavity black sources

- CNT sources on 1/8 inch thick substrates enable two sources to fit in the short dimension of a $6 \mathrm{U}$ spacecraft $(<10 \mathrm{~cm})$

- CNT sources are rugged

- Measurements on Ball CNT sources show no BRDF or visual change after thermal cycling $(-30 \mathrm{C}$ to $+50 \mathrm{C})$

- Almost no particulates after vibration testing 


\section{The measured emissivity of CIRiS flight CNT samples is $>0.996$}

- The high emissivity contributes to high radiometric calibration accuracy in two ways:

1. Reduces error from emissivity uncertainty

2. Reduces stray light reflection during calibration $(R<0.0036)$

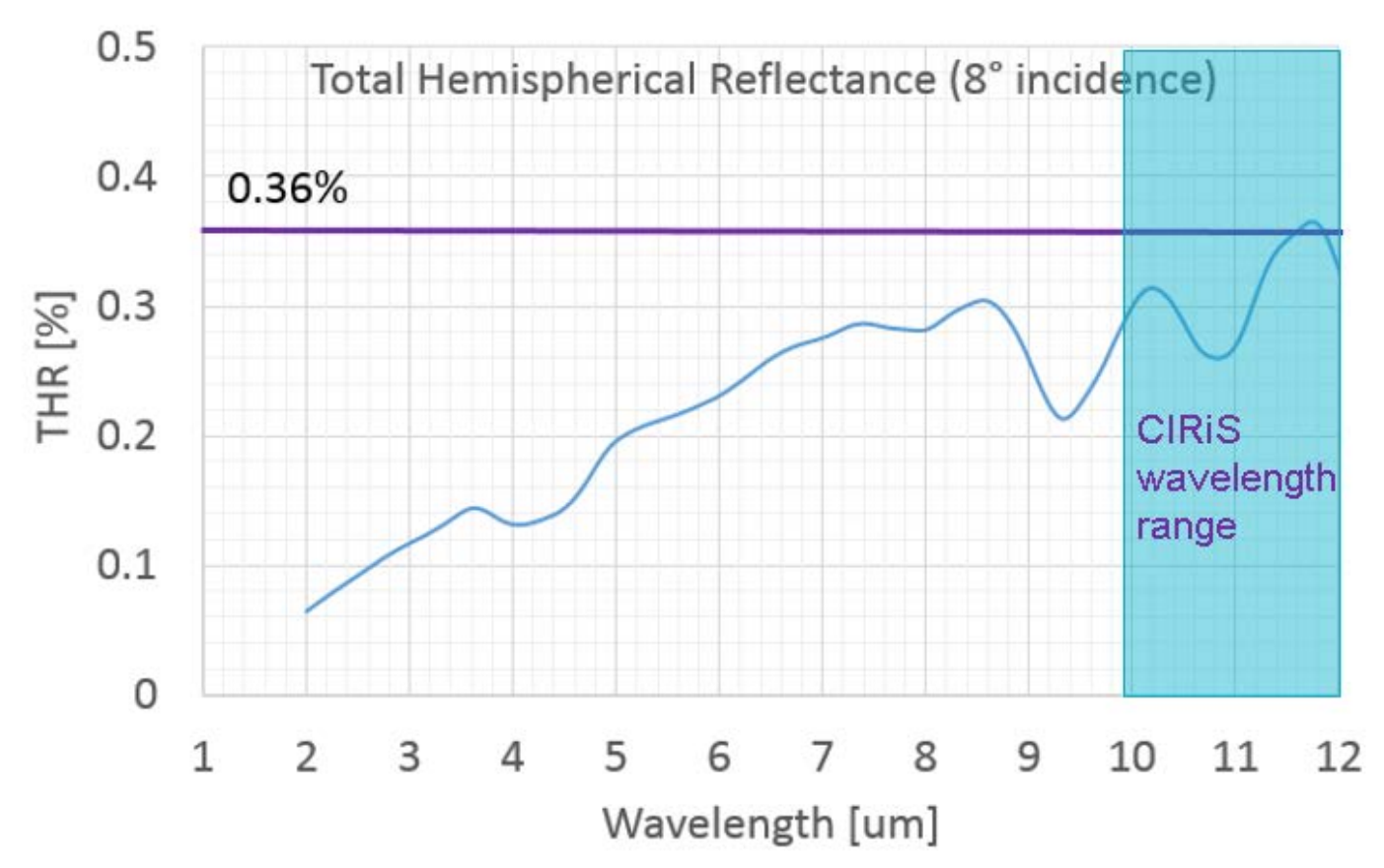

NIST measurements of a CIRiS carbon nanotube source shows reflectance $<0.36 \%$, resulting in emissivity $>0.996$ 


\section{CIRiS on-orbit radiometric accuracy is dependent on ground calibration accuracy}

- Pre-launch ground calibration procedure uses a NIST traceable blackbody source

- The CIRiS on-board CNT sources transfer the ground calibration to space

- A radiometric uncertainty model is now being developed to predict CIRiS ground and on-orbit calibration accuracy

- This procedure has been implemented for an aircraft mounted instrument (BESST) from which the CIRiS design was derived. The measured BESST calibration achieves:

- In-flight accuracy of 0.3 deg C

- In-flight precision of 0.16 deg C

- CIRiS is expected to improve on this

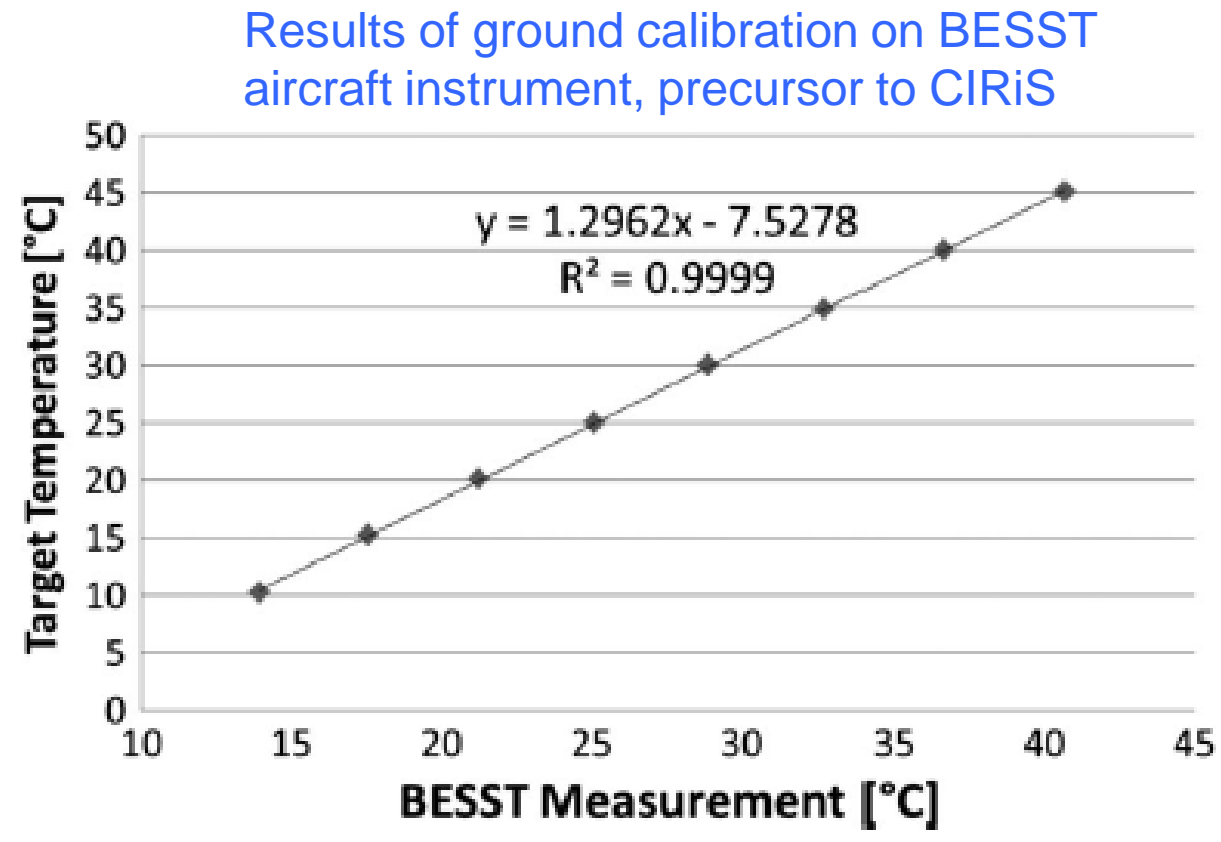




\section{The CIRIS thermal subsystem contributes to overall radiometric performance}

- Thermal control implemented in 4 separate zones

- Temperature knowledge collected from 12 sensors around instrument for additional background correction if necessary

- Thermal model for representative LEO orbits shows temperature excursions of blackbody sources and FPA housing $<+/-0.01$ deg $C$

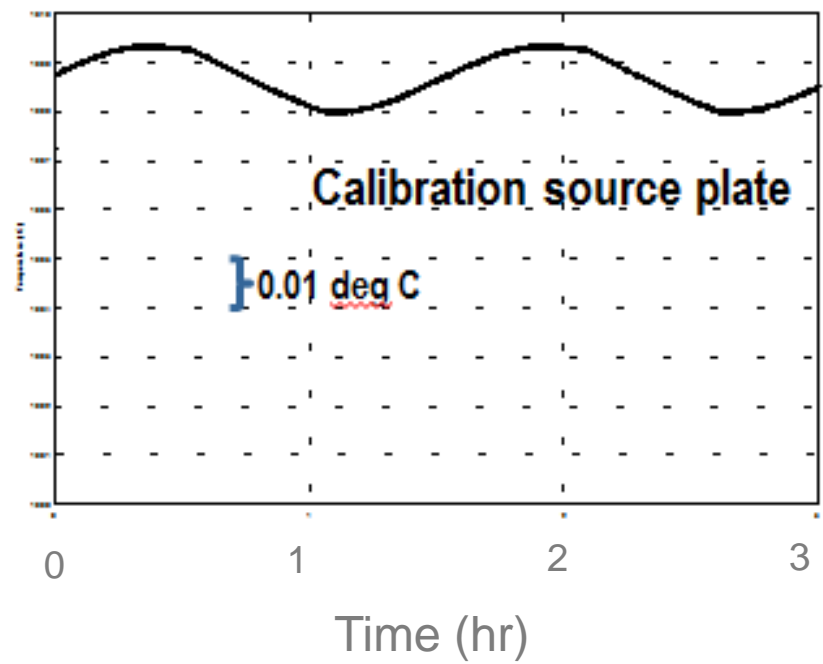

$$
\text { Upper Earth baffle }
$$$$
\text { briefly exposed to sun Time (hr) }
$$

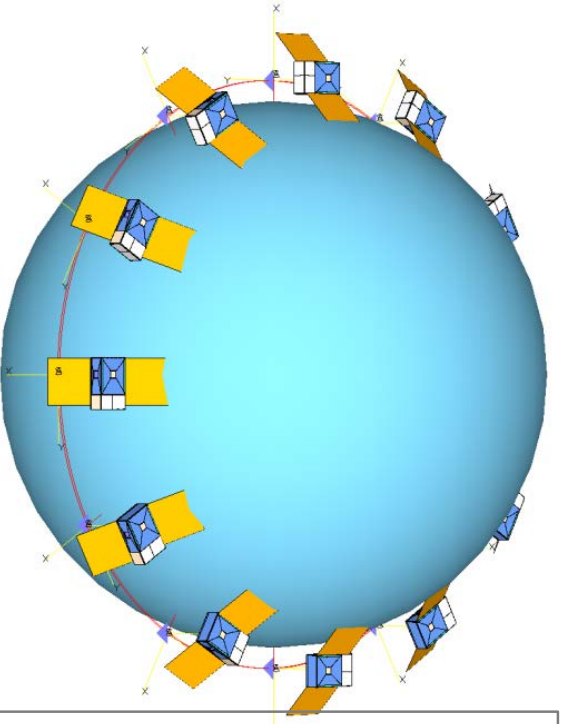

$440 \mathrm{~km}$ altitude Polar orbit, 98 degree inclination 45 degree sun beta angle 


\section{The CIRiS detector is an uncooled microbolometer FPA}

- No cryocooler or TEC necessary

- Ball has tested microbolometer FPAs from four US vendors

- FPA characterization performed for CIRiS and the E-THEMIS instrument (Europa mission/ASU) program includes radiation testing

\begin{tabular}{|l|l|}
\hline \multicolumn{2}{|c|}{ CIRiS FPA } \\
\hline Format & $640 \times 480$ \\
\hline Pixel Size & $12 \mathrm{um}$ \\
\hline Frame rate & $30 \mathrm{fps}$ or $60 \mathrm{fps}$ \\
\hline $\begin{array}{l}\text { Noise Equivalent Temp } \\
\text { Difference (NEDT) }\end{array}$ & $<50 \mathrm{mK}(\mathrm{F} / 1,290 \mathrm{~K})$ \\
\hline Volume & $26 \times 26 \times 33 \mathrm{~mm}$ \\
\hline Mass & $40 \mathrm{gm}$ \\
\hline Power & $<1 \mathrm{~W} @ 30 \mathrm{fps}$ \\
\hline
\end{tabular}

- Formats of commercial uncooled microbolometer FPAs now available up to $1024 \times 768$ format. 


\section{The CIRiS optical system is intentionally simple for the CIRiS mission technology demonstration}

- A single Ge lens with one aspheric surface for improved off-axis performance

- Low F/\# =1.8 for high SNR

- Limitation on F/\# reduction is $6 \cup$ Cubesat envelope

- The CIRiS optomechanical structure is compatible with a range of other optical designs, both refractive and reflective

\begin{tabular}{|l|l|}
\hline Parameter & Value \\
\hline F/\# & F/1.8 \\
\hline Focal Length & $36.0 \mathrm{~mm}$ \\
\hline $\begin{array}{l}\text { Entrance Pupil } \\
\text { Aperture }\end{array}$ & $20.0 \mathrm{~mm}$ \\
\hline Angular resolution & 0.00122 radians \\
\hline Field of View & $12.2 \times 9.2 \mathrm{deg}$ \\
\hline $\begin{array}{l}\text { GSD from } 400 \mathrm{~km} \\
\text { altitude (one pixel) }\end{array}$ & $0.133 \mathrm{~km}$ \\
\hline
\end{tabular}

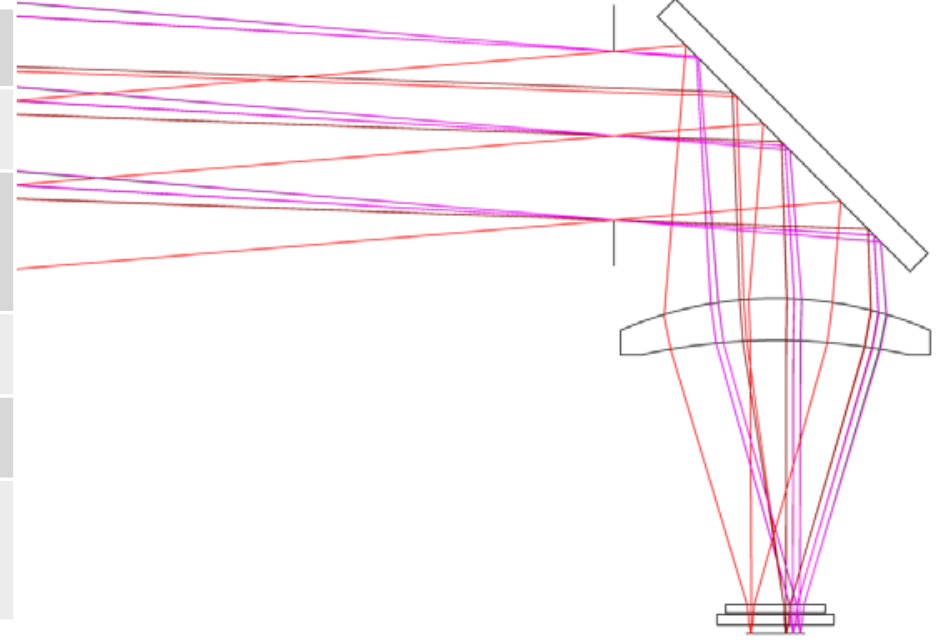

CIRiS optical system with one lens

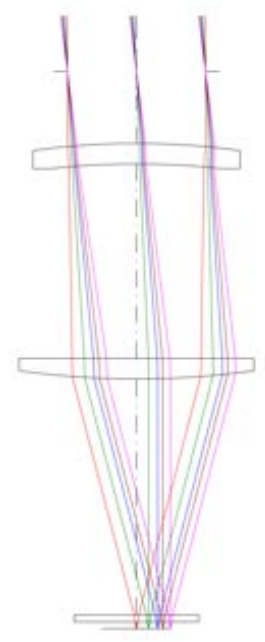

Two lens design 


\section{The butcher block filter geometry combines three dielectric filters}

- Images acquired in all three wavelength bands by pushbroom scanning

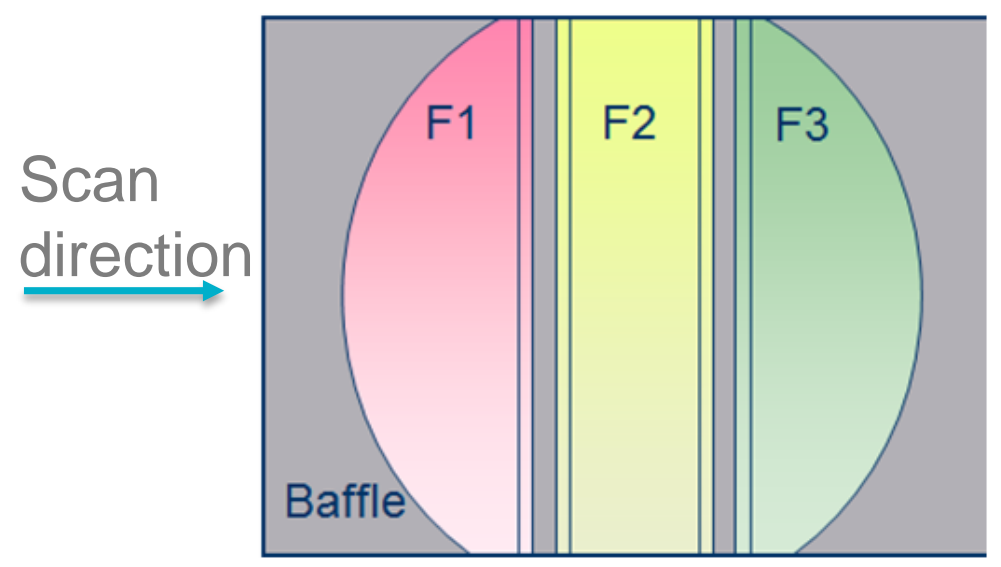

Butcher block filter assembly

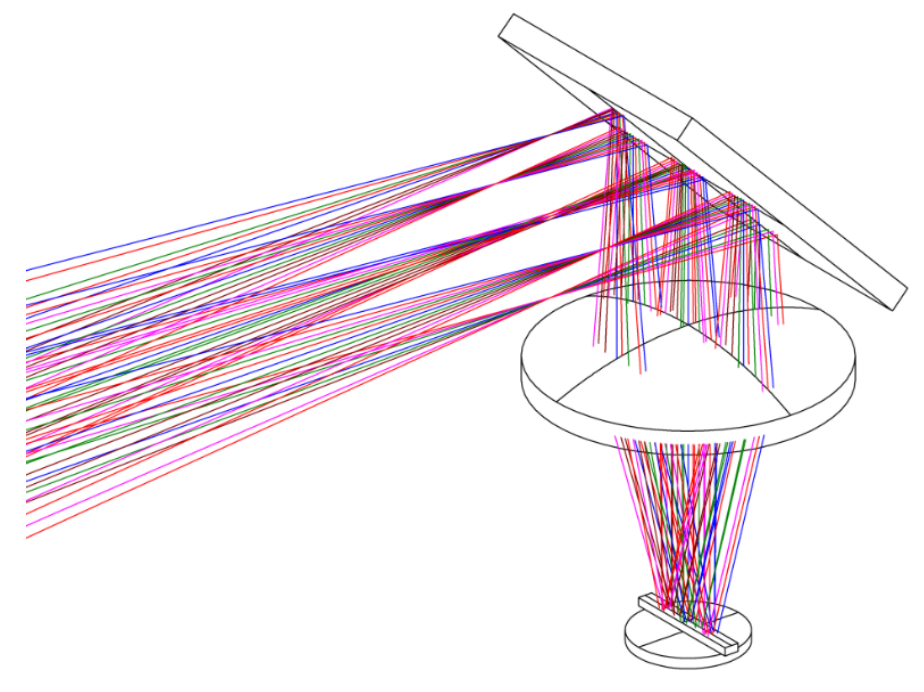

\begin{tabular}{|l|l|l|l|}
\hline Function & Band (um) & $\begin{array}{l}\text { Band pass } \\
\text { (um) }\end{array}$ & $\begin{array}{l}\text { Center } \\
\text { wavelength (um) }\end{array}$ \\
\hline $\begin{array}{l}\text { Split window band 1 } \\
\text { (atmospheric correction) }\end{array}$ & 9.85 to 11.35 & 1.5 & 10.6 \\
\hline $\begin{array}{l}\text { Split window band 2 } \\
\begin{array}{l}\text { High signal for thermal } \\
\text { imaging }\end{array}\end{array}$ & 11.77 to 12.6 & 0.91 & 12.23 \\
\hline
\end{tabular}




\section{The CIRiS on-orbit concept of operations will implement variants on a basic calibration procedure}

- Goals of calibration investigation:

- Space validation of calibration procedures

- Optimization of calibration procedures (accuracy, dynamic range, time between cals)

- Variables to be investigated:

1. Calibration views used and their order: 1,2 or 3

2. Temperature setting of heated calibration source: $280 \mathrm{~K}$ to $300 \mathrm{~K}$

3. Time between calibrations

4. Dwell time/averaging time at each calibration 


\section{CIRiS is integrated to a $6 \mathrm{U}$ CubeSat spacecraft bus}

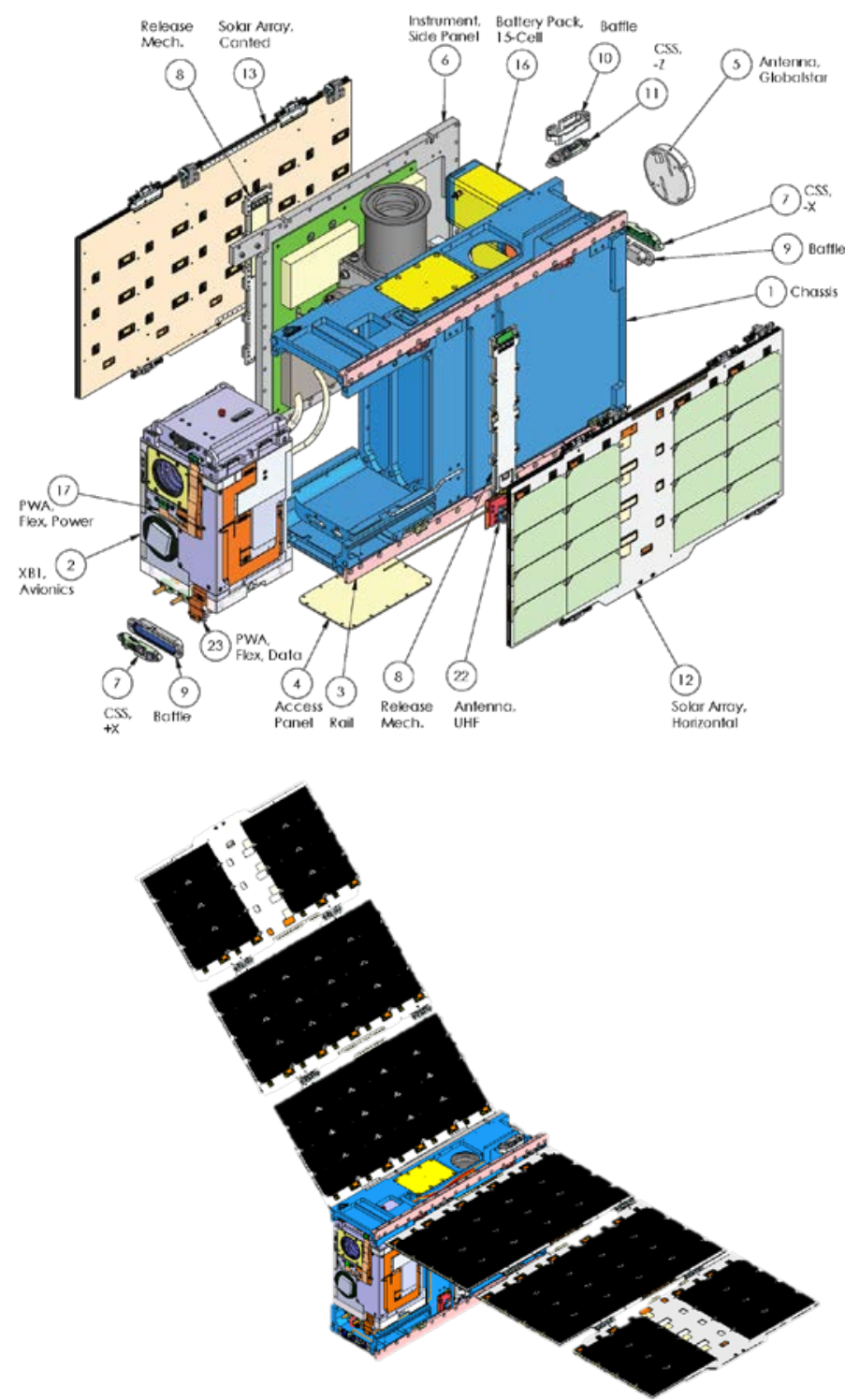

Spacecraft functions include:

- Guidance, Navigation \& Control

- 3-axis control, star tracker

- Power Subsystem

- Power distribution, solar panels, battery storage

- Spacecraft command and Data Handling

- Command control, data storage, telemetry

- RF communication

- Globalstar Radio

- Payload electrical interface

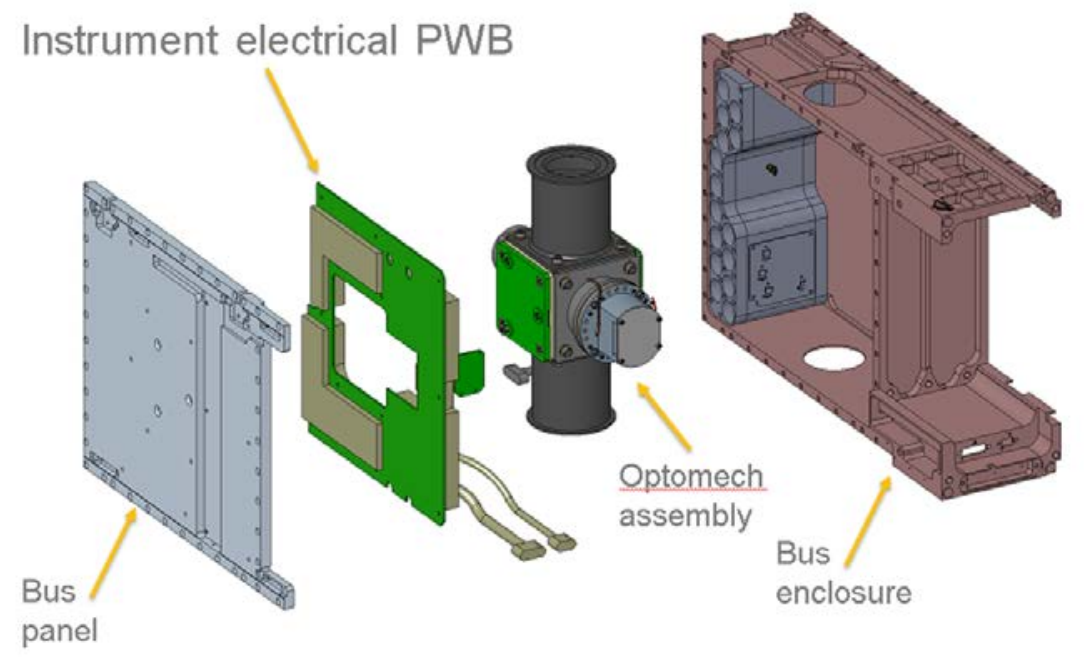




\section{Extensive testing conducted on CNT source Engineering Design Unit}

- $\quad$ Three temperature sensors embedded in EDU behind CNT substrate for nonuniformity measurement

- $\quad$ Flight temperature sensors are space-qualified; procured from another Ball space program

- EDU subjected to thermal cycling in air, thermovac, radiometric imaging

- Establishing workmanship, thermal performance, factors affecting calibration

CNT on

$1 / 8$ in

thick

substrate

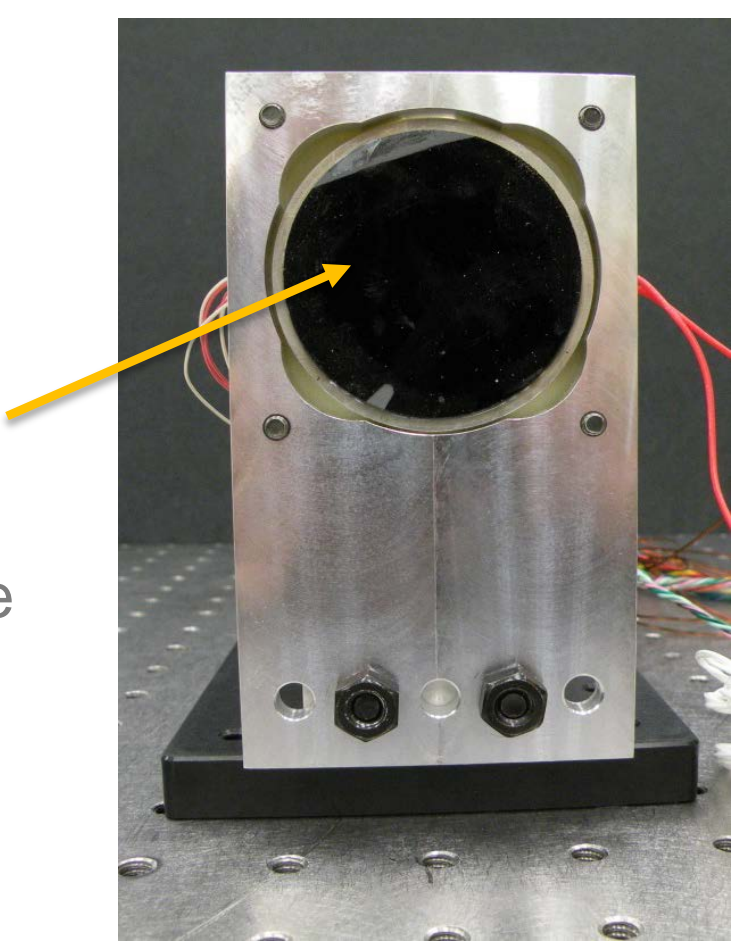


CNT calibration source EDU cycled over qualification thermal range to verify workmanship quality

- The fifth cycle went $10 \mathrm{C}$ below the cold qualification temperature

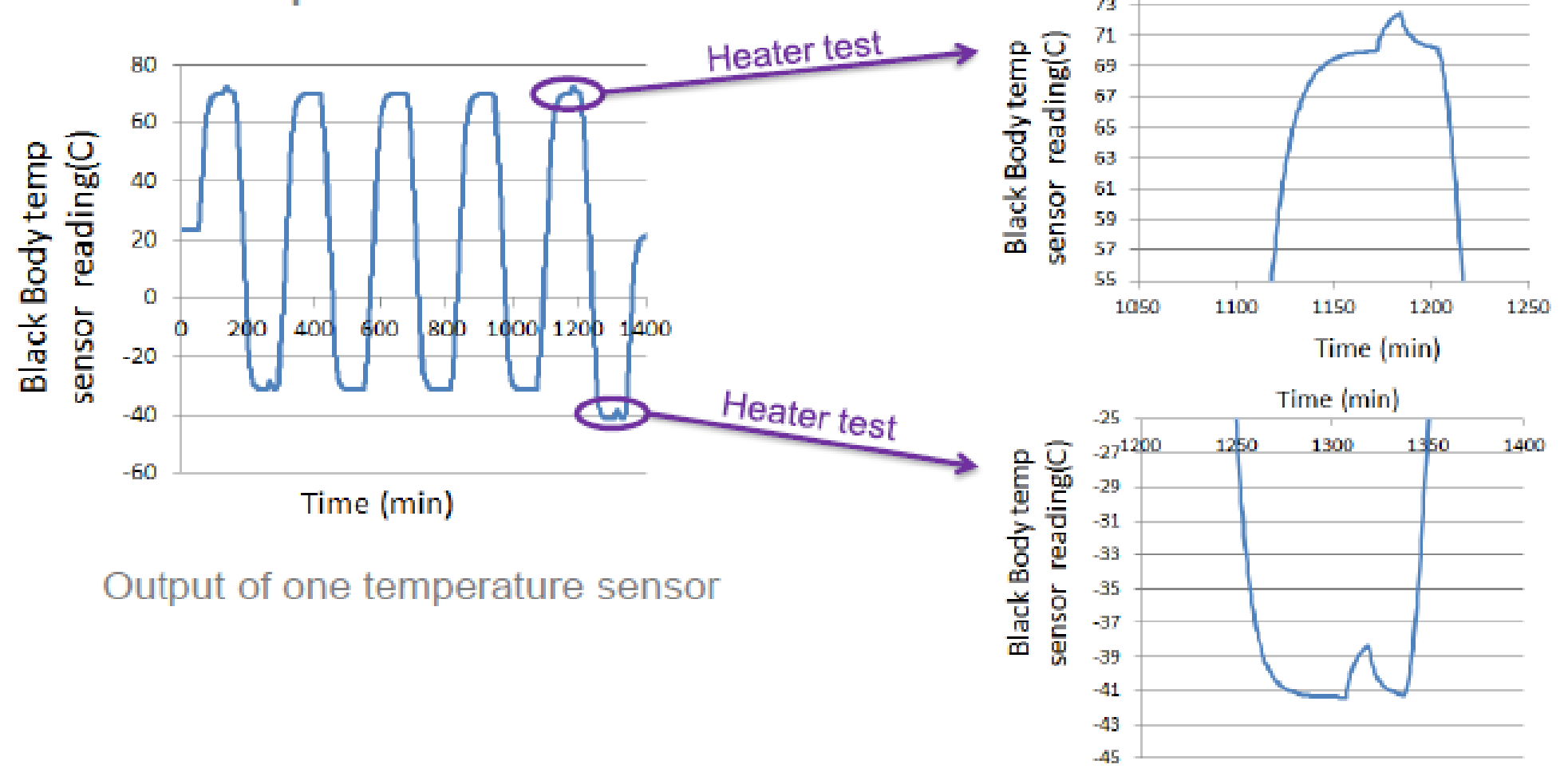




\section{CIRiS reduces size, weight and power relative to the aircraft mounted BESST}

\begin{tabular}{|l|l|l|}
\hline & BESST & CIRiS \\
\hline Weight $(\mathrm{kg})$ & 1.35 & 1.05 \\
\hline Avg power $(\mathrm{W})$ & 20 & 10 \\
\hline Envelope $\left(\mathrm{cm}^{3}\right)$ & $18 \times 19 \times 9$ & $18 \times 19 \times 9$ \\
\hline & BESST & CIRiS \\
\hline FOV & $29 \mathrm{deg} \times 22 \mathrm{deg}$ & $\begin{array}{l}12.2 \mathrm{deg} \times 9.2 \\
\text { deg }\end{array}$ \\
\hline FPA Pixel Size & $38 \mathrm{um}$ & $12 \mathrm{um}$ \\
\hline FPA Format & $324 \times 256$ & $640 \times 480$ \\
\hline FPA NEDT & $<65 \mathrm{mK}$ & $<50 \mathrm{mK}$ \\
\hline Frame rate & $4 \mathrm{~Hz}$ & $30 \mathrm{~Hz} / 60 \mathrm{~Hz}$ \\
\hline Band 1 & $10.2-10.9 \mathrm{um}$ & $9.9-11.4 \mathrm{um}$ \\
\hline Band 2 & $8.0-12.0 \mathrm{um}$ & $7.5-13.0 \mathrm{um}$ \\
\hline Band 3 & $11.3-12.1 \mathrm{um}$ & 11.8 to $12.7 \mathrm{um}$ \\
\hline
\end{tabular}




\section{CIRiS Status as of August 12017}

- All mechanical parts fabricated

- All procurements completed

- Flight CNT source assemblies fabricated

- Electronics board on-order

- Spacecraft electronics EDU delivered

- Spacecraft in functional test

- Launch anticipated late 2018; waiting to hear date 


\section{Acknowledgements}

CIRiS development is supported by the NASA ESTO InVEST (In Space Validation of Earth Science Technology) program

The Ball team:

- Alfonso Amparan

- Sandie Collins

- John Ferguson

- Bill Good

- Tom Kampe

- David Osterman

- Reuben Rohrschneider

- Bob Warden

- Partners Blue Canyon Technology (S/C) and Utah State Space Dynamics Laboratory (mission ops) 